\title{
The Relationship between Energy, Phase Charge, Impedance, and Perceived Sensation in Electrotactile Stimulation
}

\author{
Aadeel Akhtar ${ }^{1,2 *} \quad$ Brandon Boyce $^{3 \dagger} \quad$ Timothy Bretl ${ }^{3} \ddagger$ \\ ${ }^{1}$ Medical Scholars Program, ${ }^{2}$ Neuroscience Program, ${ }^{3}$ Department of Aerospace Engineering \\ University of Illinois at Urbana-Champaign
}

\begin{abstract}
Electrotactile stimulation is a common method of sensory substitution and haptic feedback. One problem with this method has been the large variability in perceived sensation that derives from changes in the impedance of the electrode-skin interface. One way to reduce this variability is to modulate stimulation parameters (current amplitude and pulse duration) in response to impedance changes, which are reflected in the time domain by changes in measured peak resistance, $R_{p}$. To work well, this approach requires knowing precisely the relationship between stimulation parameters, peak resistance, and perceived sensation. In this paper, experimental results show that at a constant level of perceived sensation there are linear relationships between $R_{p}$ and both peak pulse energy, $E_{p}$, and phase charge, $Q$, from which stimulation parameters are easily computed. These linear relationships held across different subjects, sessions, magnitudes of sensation, stimulation locations, and electrode sizes. The average $\mathrm{R}^{2}$ values for these linear relationships were 0.957 for $E_{p}$ vs. $R_{p}$ and 0.960 for $Q$ vs. $R_{p}$, indicating a nearly perfect fit.
\end{abstract}

Index Terms: H.5.2 [Information Interfaces and Representation]: User Interfaces-Haptics I/O; H.5.2 [Information Interfaces and Representation]: User Interfaces-Theory and methods;

Table 1: List of notation.

\begin{tabular}{ll}
\hline Abbreviation & Meaning \\
\hline$I$ & Current \\
$T$ & Pulse Duration \\
$V_{p}$ & Peak Voltage \\
$R_{p}$ & Peak Resistance \\
$Q$ & Phase Charge \\
$\hat{Q}$ & Desired Phase Charge \\
$E_{p}$ & Peak Pulse Energy \\
$m_{Q}$ & Slope of $Q$ vs. $R_{p}$ constant sensation line \\
$m_{E}$ & Slope of $E_{p}$ vs. $R_{p}$ constant sensation line \\
$\left(x^{*}, y^{*}\right)$ & Point of convergence of $Q$ vs. $R_{p}$ constant \\
& sensation lines \\
\hline
\end{tabular}

\section{INTRODUCTION}

Electrotactile stimulation is the application of electrical current over the skin to stimulate sensory nerves. The sensations elicited from electrotactile sensation can be felt as vibration, touch, tingling, itching, pinching, pressure, and pain, among others, by varying stimula-

*e-mail:aakhta3@illinois.edu

†e-mail:boyce4@illinois.edu

†e-mail:tbretl@illinois.edu

IEEE Haptics Symposium 2014

23-26 February, Houston, Tx, USA

U.S. Government work not protected by U.S. copyright

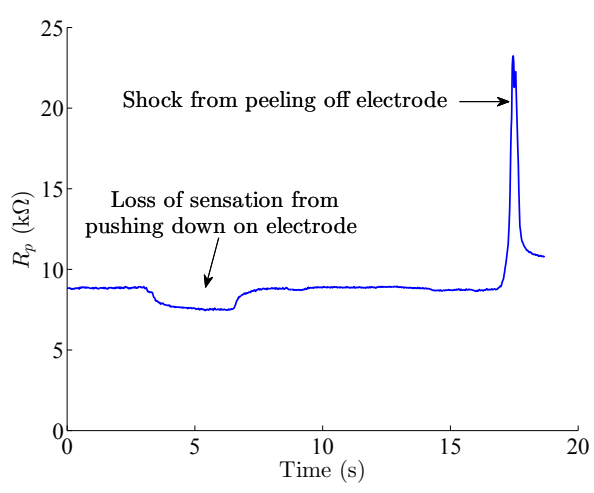

Figure 1: Changes in the peak resistance of the electrode-skin interface due to external disturbances. These changes result in a perceived loss of sensation when the peak resistance drops and an increase in sensation when the peak resistance rises.

tion parameters such as the waveform, frequency, location, or electrodes [1]. Consequently, electrotactile stimulation is often used in sensory substitution applications, where a user replaces a lost sensory modality with another sense [2]. In the case of electrotactile sensory substitution, the lost sensory modality is replaced with touch. Electrotactile stimulation is also useful as a method for delivering information via haptic feedback to a user, such as conveying texture in a multitouch display [3], suture tension to a physician teleoperating a surgical robot [4], or touch and proprioception to amputees who wear prostheses [5].

One problem with electrotactile stimulation has been the large variability in perceived sensation that derives from changes in the impedance of the electrode-skin interface. The changes in impedance may be caused by physiological disturbances, such as the accumulation of sweat, or by mechanical disturbances, such as varying contact between the electrode and the skin [1]. As an example, Fig. 1 shows impedance changes-reflected in the time domain by measurements of peak resistance - that were caused by manipulation of the electrodes. The subject in this case wore electrodes on the forearm and was stimulated with a monophasic square pulse at $3.5 \mathrm{~mA}$ with a pulse duration of $200 \mu \mathrm{s}$. The subject perceived a loss in sensation when the electrodes were pushed gently into the skin (decreasing the impedance), and perceived a gain in sensation when the electrodes were pulled away (increasing the impedance).

Efforts have been made over the past thirty years to reduce variability in perceived sensation by modulating stimulation parameters, specifically current amplitude $(I)$ and pulse duration $(T)$, in response to measurements of impedance. To work well, this approach requires knowing precisely the relationship between stimulation parameters, impedance, and perceived sensation. Existing characterizations of this relationship-although they have been used successfully to reduce variability in perceived sensation-leave room for improvement. For example, seminal work by Tachi et al. [2] 
equated a constant level of sensation with a constant pulse energy. This work was based upon the assumption that impedance is independent of current, an assumption that is known to be false $[1,6]$. More recent work by Kajimoto [7] discarded this assumption but relied on a relationship between pulse duration and impedance with a low $\mathrm{R}^{2}$ value $(0.3585)$, suggesting that results may not have been consistent across different subjects, magnitudes of sensation, and locations of stimulation. Kantor et al. [8] observed that phase charge remains nearly constant for constant sensation, but other studies — such as the one by Baker and Bowman [9] — provide evidence that phase charge can vary even while perceived sensation remains constant. Our purpose in this paper is to establish a relationship between stimulation parameters, impedance, and perceived sensation that is not based on flawed assumptions and that better matches experimental results.

In particular, we will show that at a constant level of perceived sensation there are linear relationships between peak resistance $R_{p}$ and both peak pulse energy $E_{p}$ and phase charge $Q$, from which stimulation parameters are easily computed. These relationships were verified in experiments with different subjects, sessions, magnitudes of sensation, and electrode sizes. Average $\mathrm{R}^{2}$ values were 0.957 for $E_{p}$ vs. $R_{p}$ and 0.960 for $Q$ vs. $R_{p}$, indicating a nearly perfect fit. We will conclude with a brief discussion of how these results might improve existing methods of real-time impedance feedback for electrotactile stimulation, although this improvement remains to be experimentally verified.

\section{Methods}

Five subjects, two male, three female (ages: 20-26), volunteered for the experiment. Subjects were asked to participate in two sessions held on different days with two trials being held each session. During each session, two 25 x $20 \mathrm{~mm}$ electrodes (AMBU Neuroline 710) were placed over glabrous skin on the proximal left forearm over the flexor carpi radialis muscle. The electrodes were placed $3 \mathrm{~cm}$ apart. Monophasic positive square pulses generated by an NImyDAQ (National Instruments) data acquisition device were fed to a linear isolated stimulator (BIOPAC STMISOLA) that provided a constant current stimulation to the subject. The voltage across the electrodes was also recorded by the NI-myDAQ. All data were collected and processed using the MATLAB DAQ Toolbox (MathWorks, Natick, MA). All procedures and equipment were approved by the Institutional Review Board of the University of Illinois at Urbana-Champaign.

For both trials, pulse durations $(T)$ were varied between 200$700 \mu$ s in increments of $50 \mu$ s and the current $(I)$ was adjusted until a specified sensation level was reached. This level was chosen to be the subject's sensation threshold in the first trial, and a significantly stronger magnitude of sensation in the second trial. Each trial consisted of collecting 11 data points, one for each value of $T$. Pulses were delivered at a frequency of $50 \mathrm{~Hz}$. The voltage measured across the electrodes is the time-varying response to the constant current pulse applied to the electrode-skin interface modeled by an $R C$ network [1]. The maximum peak $\left(V_{p}\right)$, as shown in Fig. 2, was averaged over 10 pulses. From this, we derive values for $R_{p}$, the peak resistance,

$$
R_{p}=\frac{\bar{V}_{p}}{I},
$$

$Q$, the phase charge for a monophasic square wave,

$$
Q=\int_{0}^{T} I \mathrm{~d} t=I T
$$

and $E_{p}$, the peak pulse energy for a monophasic square wave,

$$
E_{p}=R_{p} \int_{0}^{T} I^{2} \mathrm{~d} t=R_{p} I^{2} T
$$
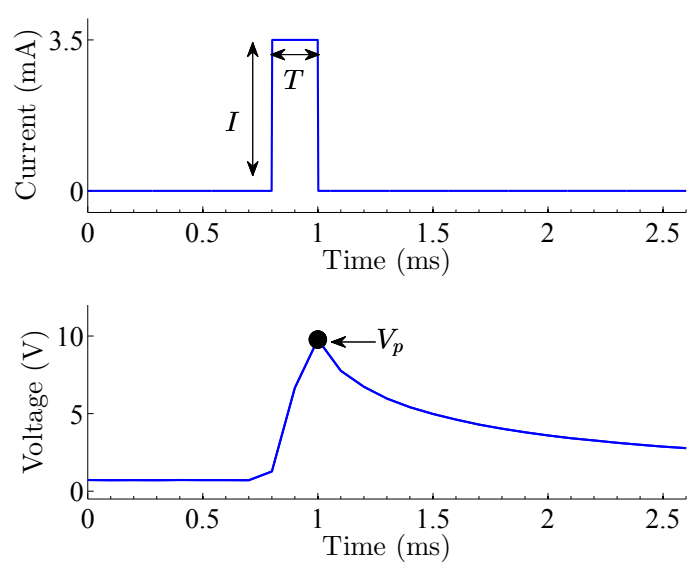

Figure 2: Current stimulation waveform (top) delivered across the skin and the resulting Voltage waveform measured (bottom). The measured voltage is the peak of the waveform $\left(V_{p}\right)$.

Each trial began by adjusting the current of a waveform with $T=$ $200 \mu$ s until the specified sensation level was reached. To ensure that each sensation level felt the same across all pulse durations, each sensation felt above $T=200 \mu$ s was compared to the initial reference sensation at $T=200 \mu \mathrm{s}$. The reference sensation would be presented to the subject for two seconds, followed by a two second period of rest before presenting the new stimulation for two seconds at a higher pulse duration. The subject would then make a decision on whether the new stimulation felt weaker, stronger, or the same as the reference stimulation. The subjects were allowed to repeat the presentation of stimulations as many times as they felt necessary to make a clear decision.

Because a range of current amplitudes may result in the same perceived sensation level, the current was increased just beyond the upper difference limen between the new sensation and the reference sensation. The current amplitude was then reduced just below this threshold to the maximum value at which the two sensations felt the same. The final current amplitude and voltage was recorded. Finally, to validate that all the sensation levels felt the same, a new reference was set at the stimulation threshold determined at $700 \mu \mathrm{s}$, and all current amplitudes and voltages at shorter pulse widths were compared again and adjusted to match the sensation felt. Since skin condition may vary daily, the subjects were asked to return for a second session later in the week and the same two trials were repeated.

Two of the subjects were selected for participating in an additional session that investigated the effect of changing the location of stimulation as well as the size of the electrodes on sensation level. This session consisted of four trials. The first two trials involved stimulation at sensation threshold of the skin lateral to the long head of the left biceps brachii muscle and the skin lateral to the right abdominal muscle. Both locations are commonly chosen stimulation sites in haptic feedback studies $[2,5,8,10,11,12]$. In the third and fourth trials, stimulation again took place on the proximal left forearm, albeit using a smaller set of electrodes sized $20 \times 15 \mathrm{~mm}$ (AMBU Neuroline 700). These trials were recorded at threshold and strong stimulation levels, respectively. All data during the additional session were collected following the same procedure as the first two sessions.

\section{RESULtS}

\subsection{Validation of the relationship between $I$ and $T$ at a constant sensation level}

In order to validate that the recorded current levels for a given pulse duration match the recorded values in previous studies, we compare 


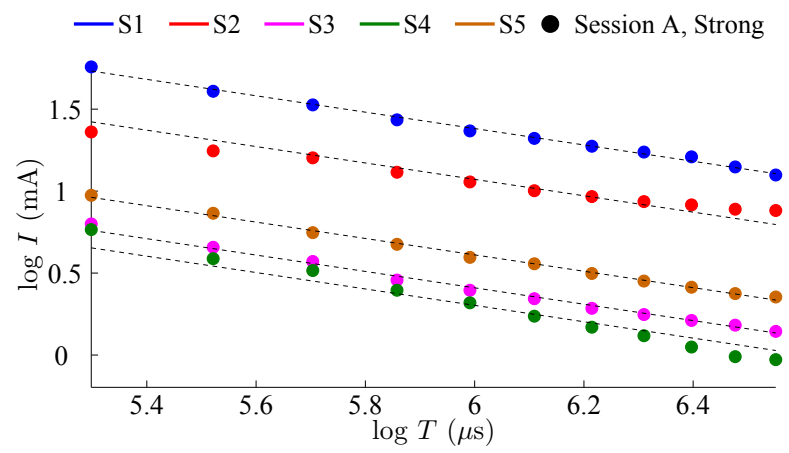

Figure 3: Current vs. Pulse Duration showing linear trends plotted on a log-log scale. For clarity, only the data from Session $A$ at the strong sensation level are shown. The best fit lines shown are constrained to a slope of -0.5 .

$I$ vs. $T$ across all subjects, sessions, magnitudes of sensation, stimulations locations, and electrode sizes. They follow the logarithmic trend mentioned in $[1,2,13]$. If we take the average of the slopes of the best fit lines of the log of the data, we obtain a value of -0.504 , which very closely matches the -0.5 slope that Tachi et al. reports in [2]. In Fig. 3, we show a subset of the data plotted with their best fit lines that have slopes constrained to -0.5 . The average $\mathrm{R}^{2}$ value of the constrained best fit lines is 0.979 , indicating a near perfect fit. Therefore, our results when comparing $I$ and $T$ are validated by their consistency with previous studies.

\subsection{Results across subjects, sessions, magnitudes of sensation, location, and electrode size}

Data showing the relationship between both $E_{p}$ vs. $R_{p}$ and $Q$ vs. $R_{p}$ across subjects, sessions, and magnitudes of sensation are plotted in Fig. 4, respectively. Each sensation felt at each data point of the same color and marker was equivalent in subjective level of sensation. All subjects across both sessions and both sensation levels showed strong linear relationships for $E_{p}$ vs. $R_{p}$ and $Q$ vs. $R_{p}$. Furthermore, when fitting best fit lines through the data, all lines tended to originate from a common point. These trends held across different stimulation locations and sizes, as shown in Fig. 5. In comparing strong vs. weak sensation magnitudes, stronger sensations had higher $E_{p}$ and $Q$ values in every case.

\subsection{Aggregate results}

All data combined over all testing conditions are shown in Fig. 6. Based on the trends of the individual best fit lines converging to a common origin point, we determine values for the points of convergence for both $E_{p}$ vs. $R_{p}$ and $Q$ vs. $R_{p}$.

Since $E_{p}=R_{p} I^{2} T$, it holds that when $R_{p}=0, E_{p}=0$. Consequently, this dictates that all linear trends between the two variables should go through the origin. Furthermore, the slopes of these lines should equal $I^{2} T$.

Because the value of $Q$ is not defined by $R_{p}$, we solve for the optimal point of convergence over all the trials. The problem can be modeled according to the following linear equation,

$$
y_{k i}-y^{*}=m_{k}^{*}\left(x_{k i}-x^{*}\right),
$$

where $k$ is the trial (line) number, $i$ is the $i$-th point within that trial, $x_{k i}$ is the $R_{p}$ value for a specific point in a trial, $y_{k i}$ is the value of $Q$ for a specific point in a trial, $\left(x^{*}, y^{*}\right)$ is the ordered pair representing the point of convergence of all the lines, and $m_{k}^{*}$ is the slope of the line for a specified trial. We solve for $m_{k}^{*}, y^{*}$, and $x^{*}$, such that the
Table 2: $R^{2}$ regression statistics aggregated across all sessions, subjects, stimulations levels, locations, and electrode sizes.

\begin{tabular}{cccccc}
\hline Parameters & Mean & Std & Min & Max & Median \\
\hline$E_{p}$ vs. $R_{p}$ & 0.957 & 0.049 & 0.764 & 0.995 & 0.975 \\
$Q$ vs. $R_{p}$ & 0.960 & 0.038 & 0.838 & 0.994 & 0.971 \\
\hline
\end{tabular}

sum squared error is minimized,

$$
\min _{m_{k}^{*}, y^{*}, x^{*}} \sum_{k} \sum_{i}\left(y_{k i}-m_{k}^{*} x_{k i}+m_{k}^{*} x^{*}-y^{*}\right)^{2} .
$$

The $m_{k}^{*} x^{*}$ term makes the objective function nonlinear, and it can be shown that the Hessian of the objective function is not positive semidefinite, which means that the function is nonconvex. As a result, we used gradient descent to find a locally optimal solution to the minimization problem. For our initial guess of $x^{*}$, the value of $R_{p}$ was found for which the differences in $Q$ between all the individual best fit lines was minimized. For the initial $y^{*}$, the average value of $Q$ was taken across all the lines evaluated at the initial $x^{*}$. Step sizes for $x^{*}, y^{*}$ and $m_{k}^{*}$ were chosen to be $10^{-7}, 10^{-5}$, and $10^{-4}$, respectively. The algorithm ran for $10^{6}$ iterations before stopping. This resulted in a point of convergence of $(-0.81 \mathrm{k} \Omega, 0.21 \mu \mathrm{C})$ for $\left(x^{*}, y^{*}\right)$. Linear regression was applied to each of the trials with the constraint that the line must go through the point of convergence.

The $\mathrm{R}^{2}$ values are reported in Table 2 . The average value for $E_{p}$ vs. $R_{p}$ was 0.957 and for $Q$ vs. $R_{p}$ was 0.960 , where an $\mathrm{R}^{2}$ value of 1.0 denotes a perfect fit to the data.

\section{Discussion}

\subsection{The linear relationship between $E_{p}$ and $R_{p}$}

Evidence for the linear relationship between $E_{p}$ and $R_{p}$ is provided both by our results and Tachi et al. [2]. The large average $\mathrm{R}^{2}(0.957)$ for $E_{p}$ vs. $R_{p}$ strongly suggests that this relationship is consistent across all subjects, sessions, magnitudes of sensation, stimulation locations, and electrode sizes. Because $E_{p}=R_{p} I^{2} T$, the slopes of the best fit lines in the $E_{p}$ vs. $R_{p}$ plot in Fig. 6 a are equivalent to $I^{2} T$. This suggests that the value of $I^{2} T$ is constant for constant levels of sensation, as determined by Tachi et al.

Though similar results were found by Tachi et al., an erroneous assumption led them to a different conclusion. Tachi et al. adjusted pulse durations of monophasic square pulses and found the amount of current needed to reach stimulation threshold for three subjects. They reported that for pulse durations under $1 \mathrm{~ms}$ there was a logarithmic trend, that when plotted on a log-log scale resulted in a linear curve with slope -0.5 . This is validated by our data as well, which when linearly fit with slopes of -0.5 achieved an $R^{2}$ of 0.979 . For a specific waveform at a constant sensation level, the relationship between $I$ and $T$ can be written as $\log I=-0.5 \log T+c$, where $c$ is a constant term. It follows that $I=c^{\prime} T^{-0.5}$, and after squaring both sides and rearranging terms, we obtain $I^{2} T=c^{\prime \prime}$. This means that for a given magnitude of sensation, the value of $I^{2} T$ always remains constant. Under the assumption that the impedance of the electrode-skin interface was constant over the course of an experimental trial, Tachi et al. reached the conclusion that the energy, $E=Z I^{2} T$, where $Z$ is the impedance of the electrode-skin interface, is also constant for a constant level of sensation. This assumption fails because it is well studied that changing $I$, as Tachi et al. did, affects the impedance significantly $[1,6]$. Therefore, for two different values of $I$, regardless of whether $I^{2} T$ is constant, $Z$ would not be the same. Furthermore, in his implementation of the constant energy controller, when the value of $Z$ changed over time, 


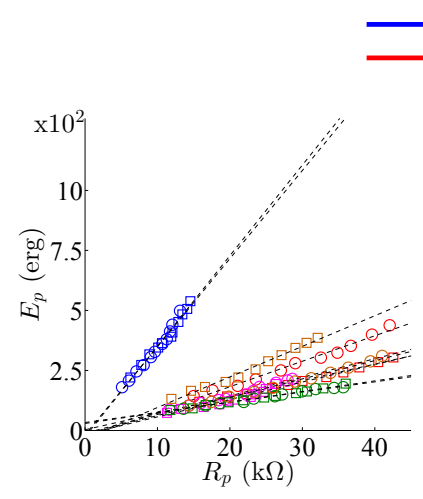

(a) $E_{p}$ vs. $R_{p}$,

A \& B, Threshold

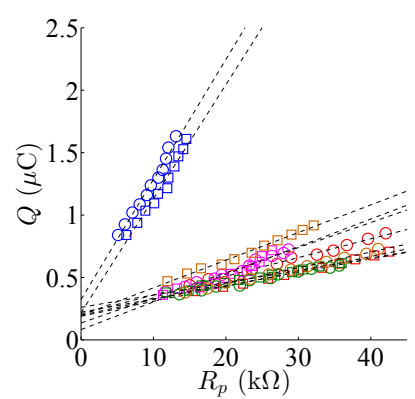

(e) $Q$ vs. $R_{p}$,

A \& B, Threshold

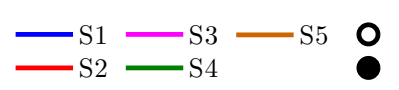

Session A, Threshold

Session A, Strong
- Session B, Threshold

Session B, Strong

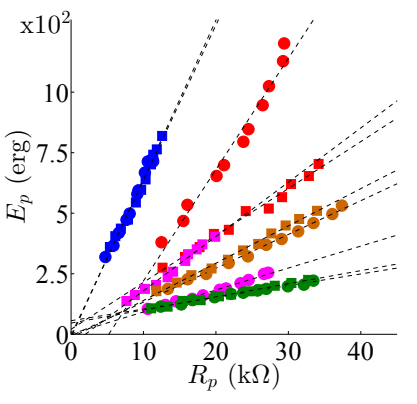

(b) $E_{p}$ vs. $R_{p}$,

A \& B, Strong

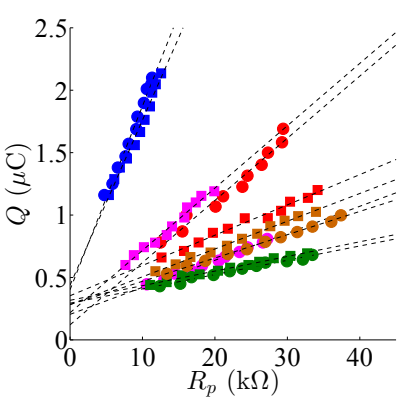

(f) $Q$ vs. $R_{p}$,

A \& B, Strong

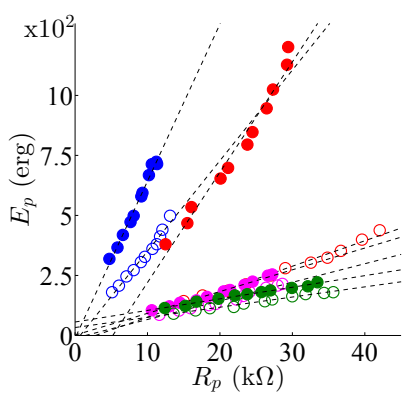

(c) $E_{p}$ vs. $R_{p}$

A, Threshold \& Strong

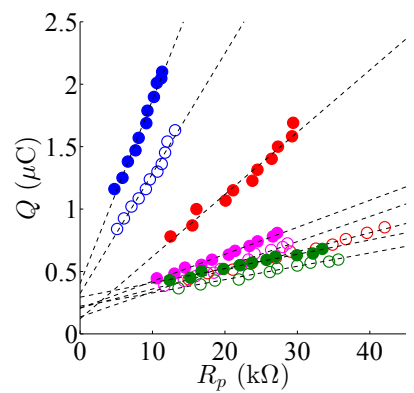

(g) $Q$ vs. $R_{p}$,

A, Threshold \& Strong

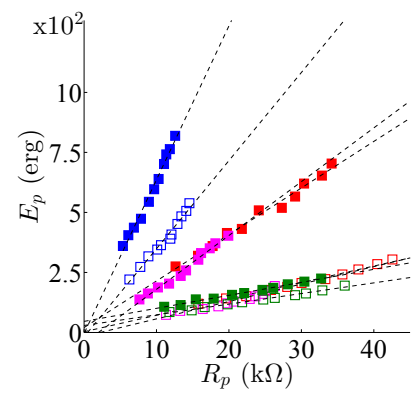

(d) $E_{p}$ vs. $R_{p}$,

B, Threshold \& Strong

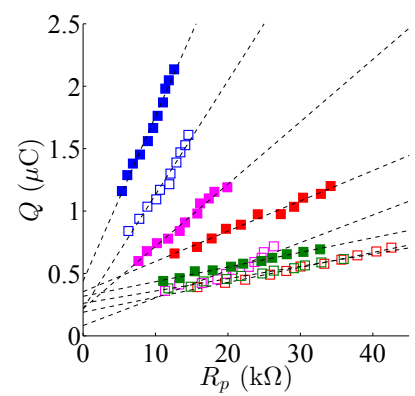

(h) $Q$ vs. $R_{p}$,

$B$, Threshold \& Strong

Figure 4: Peak Energy and Phase Charge vs. Peak Resistance plots showing linear trends across all subjects over two stimulation sessions. Sessions A and B are compared in (a) and (e) at stimulation threshold and at a strong sensation magnitude in (b) and (f). Plots (c), (d), (g), and (h) directly compare threshold vs. strong sensations for the different sessions.

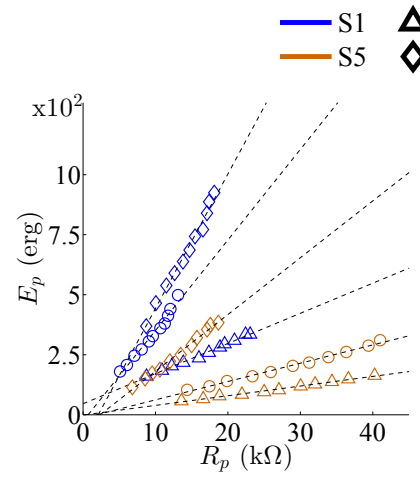

(a) $E_{p}$ vs. $R_{p}$, Threshold, Locations
$\Delta$ Bicep $\quad \mathbf{O} 25 \times 20 \mathrm{~mm}$ Threshold (Forearm)

Abdomen

$\boldsymbol{\nabla} 20 \times 15 \mathrm{~mm}$ Threshold (Forearm)

- $25 \times 20 \mathrm{~mm}$ Strong (Forearm)

$\nabla 20 \times 15 \mathrm{~mm}$ Strong (Forearm)

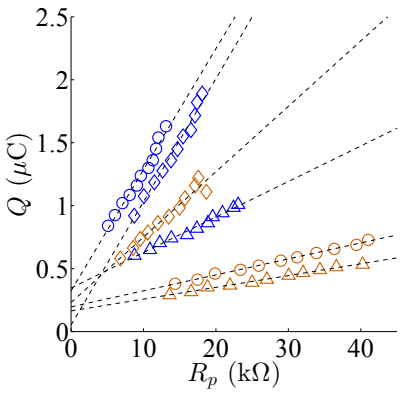

(b) $Q$ vs. $R_{p}$,

Threshold, Location

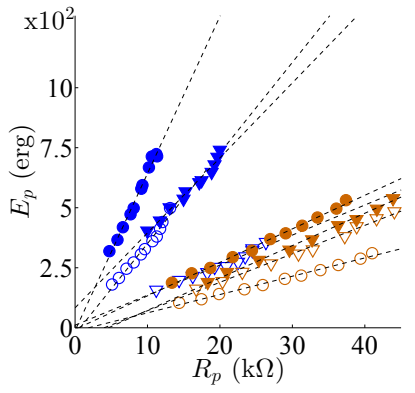

(c) $E_{p}$ vs. $R_{p}$

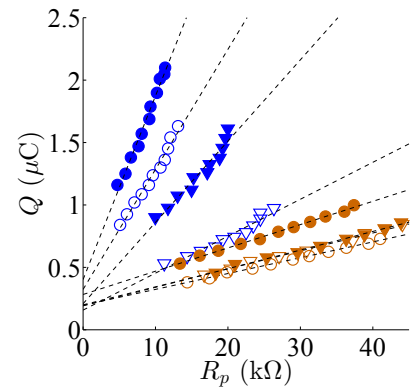

(d) $Q$ vs. $R_{p}$,
Thresh. \& Strong, Sizes Thresh. \& Strong, Sizes

Figure 5: Peak Energy (a) and Phase Charge (b) vs. Peak Resistance plots comparing multiple stimulation locations, namely the left forearm, left bicep, and right abdomen. Peak Energy (c) and Phase Charge (d) vs. Peak Resistance plots comparing two electrode sizes. Note the linear trends seen across experimental trials similar to Fig. 4 . 

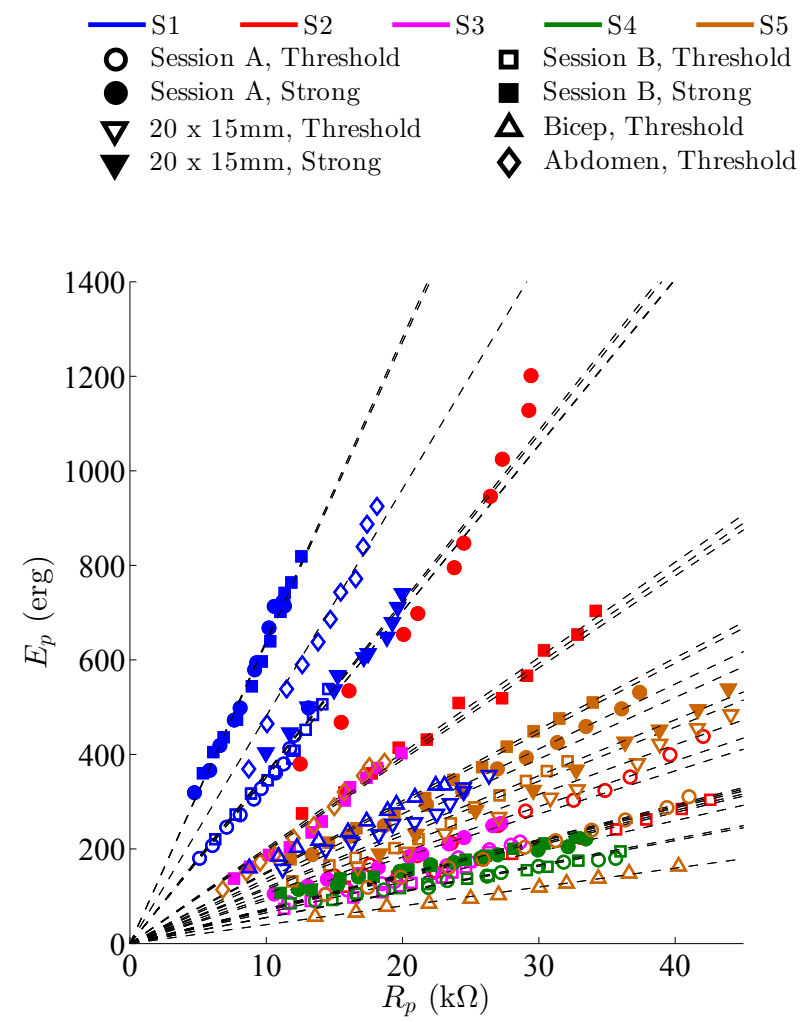

(a)

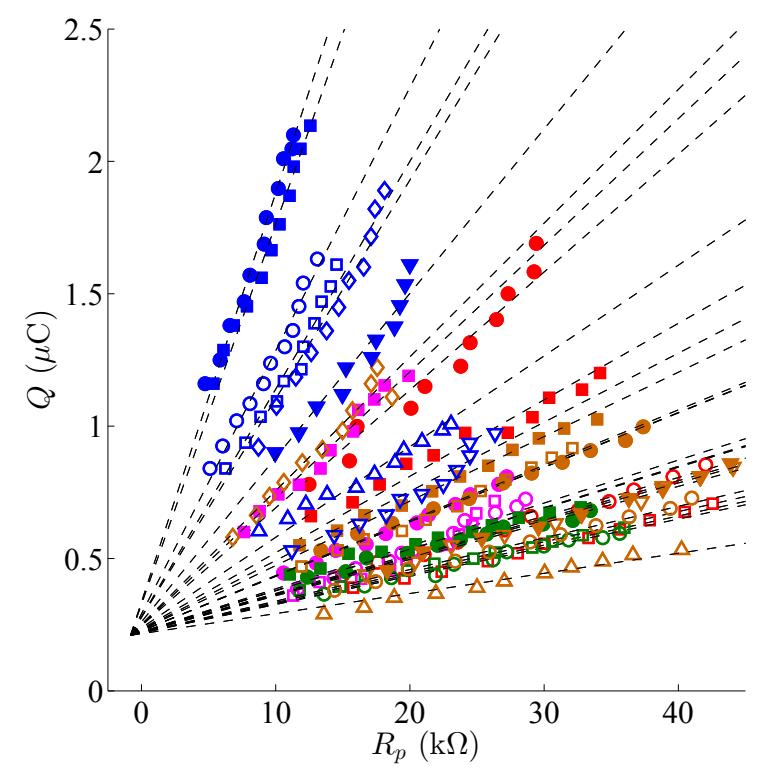

(b)

Figure 6: Peak Energy (a) and Phase Charge (b) vs. Peak Resistance plots aggregating all trials across different sessions, subjects, sensation magnitudes, stimulation locations, and electrode sizes. Best fit lines were constrained to go through the origin in (a), while all of the lines in (b) were constrained to go through an optimal point of convergence $(-0.81 \mathrm{k} \Omega, 0.21 \mu \mathrm{C})$. Each best fit line represents a line of constant sensation.
$I$ would be adjusted to maintain a constant $E$ for a fixed $T$. Consequently, the value of $I^{2} T$ will always be changing while trying to maintain a constant level of sensation. This contradicts the notion that $I^{2} T$ must be held constant in order to maintain a constant level of sensation, as evidenced by the data of Tachi et al. as well as ours.

\subsection{The linear relationship between $Q$ and $R_{p}$}

Similar to the relationship between $E_{p}$ and $R_{p}$, the large average $\mathrm{R}^{2}(0.960)$ for $Q$ vs. $R_{p}$ provides strong evidence of a linear relationship between the variables that is consistent across all subjects, sessions, magnitudes of sensation, stimulation locations, and electrode sizes. However, while the definition of $E_{p}$ mandates that the point of convergence of the $E_{p}$ vs. $R_{p}$ constant sensation lines is at the origin, it is unknown why the point of convergence of the $Q$ vs. $R_{p}$ constant sensation lines is approximately $(-0.81 \mathrm{k} \Omega, 0.21 \mu \mathrm{C})$. Future work will investigate a physiological model that may explain this behavior.

The linear relationship we have found between $Q$ and $R_{p}$ is critical to computing stimulation parameters for constant sensation in response to impedance changes, though it has not been considered in previous studies. As previously mentioned, the value of $I^{2} T$ should be constant in order to maintain a constant sensation level. However, simply holding this value constant is not sufficient because it does not account for changes in impedance. Recall the example in Fig. 1 and Sec. 1. In this example, $I$ and $T$ were held fixed, thus making $I^{2} T$ constant. Nevertheless, changes in sensationcorresponding to the changes in impedance shown-were still felt in light of $I^{2} T$ being constant. Consequently, an additional relationship between the stimulation parameters $I$ and $T$ and impedance is necessary. Because $Q=I T$, the linear relationship between $Q$ and $R_{p}$ satisfies this requirement. We show how to compute $I$ and $T$ from the linear relationships between $E_{p}, Q$ and $R_{p}$ in Section 4.3.

\subsection{Computing $I$ and $T$ from the linear relationships be- tween $E_{p}, Q$, and $R_{p}$}

Using the following two equations based off of the linear relationships found, we can solve for explicit values of $I$ and $T$ that hold sensation level constant and take $R_{p}$ into account,

$$
m_{E}=I^{2} T
$$

where $m_{E}$ is the slope of the constant sensation line from $E_{p}$ vs. $R_{p}$ and is independent of $R_{p}$, and

$$
\hat{Q}=m_{Q}\left(R_{p}-x^{*}\right)+y^{*}=I T,
$$

where $\hat{Q}$ is the desired $Q$ determined from the $Q$ vs. $R_{p}$ constant sensation line, $m_{Q}$ is the slope of the constant sensation line, and $\left(x^{*}, y^{*}\right)$ is the point of convergence of all constant sensation lines for $Q$ vs. $R_{p}$. The values of $m_{E}$ and $m_{Q}$ would be chosen a priori, corresponding to a desired sensation level. Rearranging these equations, we obtain

$$
I=\frac{m_{E}}{\hat{Q}}, \quad T=\frac{\hat{Q}^{2}}{m_{E}} .
$$

Figure 7 shows how these results might be used to regulate sensation level by modulating stimulation parameters at each pulse in response to measurements of peak resistance. Again, recall the example in Fig. 1 and Sec. 1. The shock recorded occurred over a duration of $500 \mathrm{~ms}$. Because the time scale of the proposed control loop occurs on a pulse-by-pulse basis, the controller should be able to prevent a shock from occurring in at most the time required for a single pulse duration (in this case, $200 \mu$ s). This approach remains to be experimentally validated and leaves open questions such as how to choose the mapping from sensation level to $m_{E}$ and $m_{Q}$. 


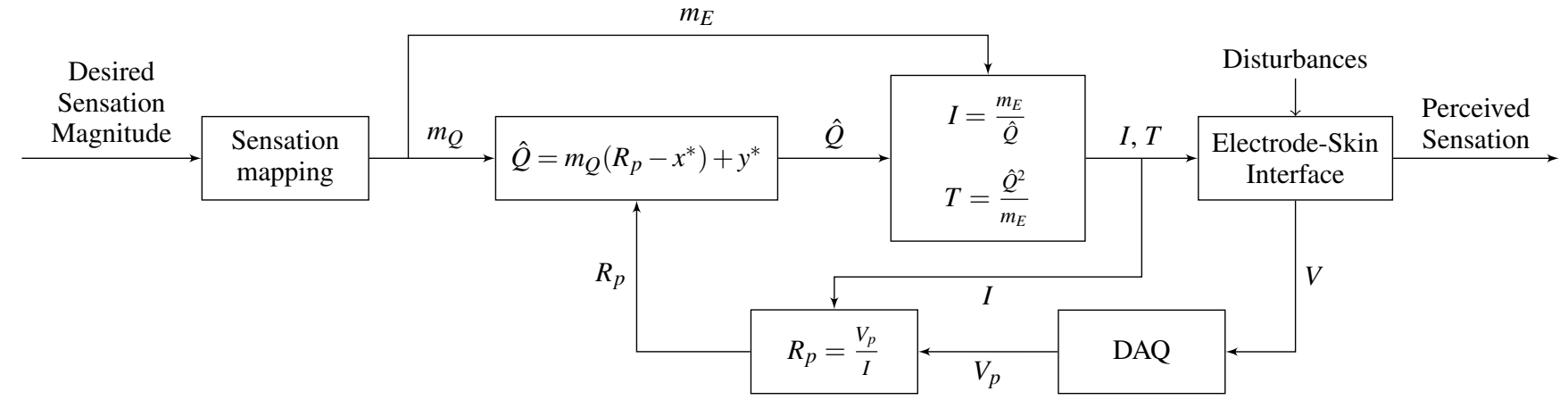

Figure 7: Block diagram for proposed control system to modulate sensation level. First, a sensation level is chosen that is mapped to $m_{E}$, equal to $I^{2} T$ and the slope of the constant sensation line that relates $E_{p}$ and $R_{p}$, and $m_{Q}$, the slope of the constant sensation line that relates $Q$ and $R_{p}$. $\hat{Q}$ represents the desired value of $Q$ from the constant sensation line of $Q$ vs. $R_{p}$, and is determined by the value of $R_{p}$ at the previous timestep. The values of $x^{*}$ and $y^{*}$ correspond to the optimal point of convergence for all $Q$ vs. $R_{p}$ constant sensation lines. From $m_{E}$ and $\hat{Q}$ the current $(I)$ and pulse duration $(T)$ used for stimulation are determined, and the appropriate waveform is delivered to the subject. The voltage ( $V$ ) is measured by the DAQ, whose peak value $\left(V_{p}\right)$ is taken and divided by $I$ to obtain the peak resistance $\left(R_{p}\right)$.

\section{Conclusion}

The use of electrotactile stimulation is common in sensory substitution and haptic feedback applications. However, physiological and mechanical disturbances cause changes in the impedance of the electrode-skin interface, thereby causing variations in the perceived sensation level. Therefore, changes in impedance, which are reflected in the time domain by changes in $R_{p}$, must be accounted for in order to maintain a constant sensation level. In examining the effects of stimulation parameters on constant sensation, we found linear relationships between $E_{p}$ and $R_{p}$, as well as $Q$ and $R_{p}$. These linear relationships held across different subjects, sessions, magnitudes of sensation, stimulation locations, and electrode sizes. Furthermore, we determined that there is a common convergence point among all best fit lines of constant sensation in both $E_{p}$ vs. $R_{p}$ and $Q$ vs. $R_{p}$. Fitting best fit lines constrained to these points resulted in average $\mathrm{R}^{2}$ values of 0.957 and 0.960 , respectively. From the relationship of $E_{p}$ vs. $R_{p}$ and $I$ vs. $T$, we verified that $I^{2} T$ is constant for constant levels of sensation, as previously determined by Tachi et al. However, we have shown that holding $I^{2} T$ constant cannot alone account for changes in sensation due to varying $R_{p}$, but the linear relationship we present between $Q$ vs. $R_{p}$ can be used in conjunction to maintain a constant sensation level. Using this information we are able to compute the stimulation parameters $I$ and $T$ for a constant sensation level while taking impedance changes into account. A control system that uses the linear relationships presented in order to modulate sensation level was proposed and will be implemented and evaluated in future work.

\section{ACKNOWLEDGEMENTS}

The authors wish to thank Navid Aghasadeghi for his help in implementing regression models and David Jun for useful discussion.

\section{RefERENCES}

[1] K.A. Kaczmarek, J.G. Webster, Paul Bach-y Rita, and Willis J. Tompkins. Electrotactile and vibrotactile displays for sensory substitution systems. Biomedical Engineering, IEEE Transactions on, 38(1):1-16, 1991.

[2] S. Tachi, K. Tanie, K. Komoriya, and Minoru Abe. Electrocutaneous communication in a guide dog robot (meldog). Biomedical Engineering, IEEE Transactions on, BME-32(7):461-469, 1985.

[3] M.E. Altinsoy and S. Merchel. Electrotactile feedback for handheld devices with touch screen and simulation of roughness. Haptics, IEEE Transactions on, 5(1):6-13, 2012.

[4] M. Kitagawa, D. Dokko, A. M. Okamura, and D. D. Yuh. Effect of sensory substitution on suture-manipulation forces for robotic surgi- cal systems. The Journal of Thoracic and Cardiovascular Surgery, 129(1): 151 - 158, 2005.

[5] H.J.B. Witteveen, E.A. Droog, J.S. Rietman, and P.H. Veltink. Vibroand electrotactile user feedback on hand opening for myoelectric forearm prostheses. Biomedical Engineering, IEEE Transactions on, 59(8):2219-2226, 2012.

[6] A. Boxtel. Skin resistance during square-wave electrical pulses of 1 to $10 \mathrm{ma}$. Medical and Biological Engineering and Computing, 15(6):679-687, 1977.

[7] H. Kajimoto. Electrotactile display with real-time impedance feedback using pulse width modulation. Haptics, IEEE Transactions on, 5(2):184-188, 2012.

[8] G. Kantor, G. Alon, and H. S. Ho. The effects of selected stimulus waveforms on pulse and phase characteristics at sensory and motor thresholds. Physical Therapy, 74(10):951-962, 1994.

[9] B. R. Bowman and L. L. Baker. Effects of waveform parameters on comfort during transcutaneous neuromuscular electrical stimulation. Annals of Biomedical Engineering, 13(1):59-74, 1985.

[10] R.W. Mann and S.D. Reimers. Kinesthetic sensing for the emg controlled "boston arm". Man-Machine Systems, IEEE Transactions on, 11(1):110-115, 1970.

[11] J. Wheeler, K. Bark, J. Savall, and Mark Cutkosky. Investigation of rotational skin stretch for proprioceptive feedback with application to myoelectric systems. Neural Systems and Rehabilitation Engineering, IEEE Transactions on, 18(1):58-66, 2010.

[12] K.A. Kaczmarek. Electrotactile adaptation on the abdomen: preliminary results. Rehabilitation Engineering, IEEE Transactions on, 8(4):499-505, 2000.

[13] E. A. Pfeiffer. Electrical stimulation of sensory nerves with skin electrodes for research, diagnosis, communication and behavioral conditioning: A survey. Medical and biological engineering, 6(6):637-651, 1968. 